\title{
Predictors that Influence Election of Contralateral Prophylactic Mastectomy among Women with Ductal Carcinoma in Situ who are BRCA-Negative
}

Nisreen Elsayegh ${ }^{1}$, Jessica Profato ${ }^{1}$, Angelica M. Gutierrez Barrera ${ }^{2}$, Heather Lin ${ }^{3}$, Henry M. Kuerer ${ }^{4}$, Can Ardic $^{2}$, Jennifer K. Litton², Debasish Tripathy², Banu K. Arun ${ }^{1,2} \bowtie$

1. Clinical Cancer Genetics, the University of Texas MD Anderson Cancer Center, Houston, TX

2. Department of Breast Medical Oncology, the University of Texas MD Anderson Cancer Center, Houston, TX

3. Department of Biostatistics, the University of Texas MD Anderson Cancer Center, Houston, TX

4. Department of Surgical Oncology, the University of Texas MD Anderson Cancer Center, Houston, TX

\begin{abstract}
$\triangle$ Corresponding author: Banu K. Arun, MD, Department of Breast Medical Oncology, The University of Texas MD Anderson Cancer Center, 1515 Holcombe Boulevard, Unit 1354, Houston, TX 77030-4009. e-mail: barun@mdanderson.org; Tel: (713) 792-2817; Fax: (713) $794-4385$
\end{abstract}

(C) 2015 Ivyspring International Publisher. Reproduction is permitted for personal, noncommercial use, provided that the article is in whole, unmodified, and properly cited. See http://ivyspring.com/terms for terms and conditions.

Received: 2015.01.27; Accepted: 2015.05.04; Published: 2015.05.23

\begin{abstract}
The authors retrospectively examined the contralateral prophylactic mastectomy (CPM) rate among 100 women with ductal carcinoma in situ who are BRCA negative. Of 100 women with ductal carcinoma in situ, 31 elected contralateral prophylactic mastectomy (CPM). Factors associated with increased likelihood of undergoing contralateral prophylactic mastectomy (CPM) among this cohort were: family history of ovarian cancer, marital status, reconstruction, mastectomy of the affected breast, and tamoxifen use.
\end{abstract}

Key words: contralateral prophylactic mastectomy, BRCA negative

\section{Introduction}

Ductal carcinoma in situ (DCIS) increases the risk for developing contralateral breast cancer (CBC). ${ }^{1}$ The risk for developing either invasive cancer or DCIS in the contralateral breast has been estimated at $0.6 \%$ per year. ${ }^{1,2}$ Increasing numbers of women with DCIS are electing contralateral prophylactic mastectomy $(\mathrm{CPM})^{2,3}$ with studies indicating a four-to five-fold increase in CPM in recent years. ${ }^{4}$ In a study of 2,037 DCIS patients, $37 \%$ (<40 years) elected CPM, and $82 \%$ ( $<40$ years) had immediate reconstruction. ${ }^{3}$ Additionally, Ashfaq et al. found that a significantly higher proportion of patients undergoing CPM had reconstruction performed $(46 \%)$ than those who did not elect CPM $(15 \%){ }^{5}$

Another significant predictor of CPM among patients with DCIS is having a germline BRCA muta- tion. ${ }^{3}$ Defining BRCA1 and BRCA2 mutation carrier status is crucial, because mutation carriers have a $43 \%$ to $84 \%$ risk of developing breast cancer (BC) and up to a $65 \%$ risk for $C B C .6,7$ The prevalence of BRCA mutations in patients with DCIS has been reported..$^{8,9}$ Our previous study ${ }^{8}$ indicated a $27 \%$ prevalence of deleterious $B R C A$ mutations among 118 patients with DCIS who were referred for genetic counseling.

We also recently reported a $27 \%$ CPM rate among 165 patients with DCIS who were evaluated for BRCA genetic testing (positive, negative, not tested). Predictors of CPM among that group were age 45 years or younger, $B R C A$ positivity, and having one or more relatives with ovarian cancer (OC). ${ }^{3}$

Although a positive $B R C A$ result has previously been shown to be associated with an increased likeli- 
hood of CPM election, our data also incidentally found a relatively high CPM rate for the $B R C A$-negative group. Among women with DCIS and a negative $B R C A$ result, $25 \%$ elected CPM. ${ }^{3}$ This relatively high rate warranted further investigation to elucidate possible predictive factors of CPM in this specific group. It has been reported that women testing negative for a $B R C A$ mutation and with a family history of $B C(\geq 2 B C$ under age 50 , or $\geq 3 B C$ at any age) have almost a four-fold increased risk of $B C .{ }^{10}$

While there is significant literature examining factors predicting CPM, there is scant literature related to the rate of CPM among this specific cohort of $B R C A$-negative patients with DCIS. Howard-McNatt et al. ${ }^{11}$ examined CPM decisions among $B R C A$-negative patients with $\mathrm{BC}$, but their study did not distinguish DCIS from invasive $\mathrm{BC}$. Among the $B R C A$-negative women with $B C$ in their study, $37 \%$ chose CPM. ${ }^{11}$

In the current study, we further evaluated factors associated with CPM in patients with DCIS who tested negative for BRCA mutation. We specifically sought to identify differences between BRCA-negative patients who underwent CPM and those who did not.

\section{Patients and methods}

One hundred women who had a diagnosis of DCIS between 2005 and 2013 underwent genetic counseling and testing and were offered an opportunity to enroll in a registry study approved by the institutional review board at The University of Texas MD Anderson Cancer Center. Each patient underwent genetic testing and received pre-test genetic counseling per standard of care. Subsequent to testing, all results were disclosed by a genetic counselor. This retrospective review included only patients with DCIS who had negative test results for BRCA1 and $B R C A 2$. We excluded patients with bilateral DCIS, $\mathrm{OC}$, micro-invasion, positive or variant of uncertain significance genetic test results, and patients who did not undergo genetic testing.

DCIS diagnoses were based on pathologic evaluation by breast pathologists at MD Anderson Cancer Center. All patients underwent genetic counseling and testing that included detailed assessment of family history. Genetic testing included comprehensive $B R C A 1$ and BRCA2 gene sequencing and, when possible, large rearrangement testing (BART).

Using the electronic patient medical records, we collected demographic and clinical information. Variables included in the analysis were age at diagnosis, race, ethnicity (Ashkenazi Jewish ancestry or not), marital status, educational level, family history of BC and/or OC in at least one first-degree relative, total number of relatives with $\mathrm{BC}$ and/or $\mathrm{OC}$, patient's $B R C A 1$ and BRCA2 test results, tumor nuclear grade (as defined by the modified nuclear grade system) estrogen receptor and progesterone receptor status (as determined by immunohistochemical analysis), surgical treatment, mastectomy of the affected breast, CPM, tamoxifen use, and reconstruction. Patient demographic and clinical characteristics were compared between the two groups defined according to CPM status. Univariate analyses were performed to determine the significance of each variable in relation to whether or not a patient had CPM, using chi-square tests for categorical variables and t-tests/ANOVA or the counterpart nonparametric approaches (Wilcoxon rank-sum or Kruskal-Wallis) for continuous variables. ${ }^{12}$ Logistic regression analysis was used to assess multivariate relationships between patient demographic and clinical characteristics and CPM.13 A logistic regression model was obtained by first including an initial set of candidate predictor variables with $p$-value $\leq 0.05$ in the univariate analysis. Stepwise backward elimination was then performed using 0.05 for the significance level of the Wald chi-square for an effect to stay in the model. Firth's penalized maximum likelihood method was used in the cases of separability.

\section{Results}

Table 1 outlines demographic and clinical characteristics. Of the 100 patients with DCIS who had negative BRCA genetic test results, 31 elected CPM and 69 did not. Sixty seven patients underwent mastectomy for the affected breast and 31 underwent only breast conservation surgery, (unknown for two patients).

Univariate analysis (Table 2) showed that married patients were more likely to elect CPM than those who were not married $(p=0.0235)$. Patients with a first-degree relative with $\mathrm{OC}$ were more likely to choose CPM than those without such a relative ( $p=$ 0.0278). Race, ethnicity, age at diagnosis, education, stage, and biomarker status were not significantly associated with CPM election.

Moreover, patients who had any relatives with OC were more likely to choose CPM than those without any relatives with OC $(p=0.0425)$; however, when analyzed by family history of BC (first-degree relative or total number), the differences were not statistically significant $(p=0.2436$ and 0.7198 , respectively). Patients receiving tamoxifen were less likely to choose CPM than those who were not $(p=0.0054)$.

Patients who underwent mastectomy were more likely to choose CPM than those who did not undergo mastectomy of the affected breast $(p<.0001)$. Finally, patients who underwent reconstruction were more 
likely to choose to have CPM than those who did not $(p<.0001)$.

Reconstruction was not included in the final multivariate regression since reconstruction (immediate vs. delayed) was only a variable for those patients who had mastectomy. When family history of OC in first-degree relatives, marital status, mastec- tomy, and tamoxifen use were included in the logistic regression model building procedure only mastectomy remained in the model; patients who underwent mastectomy were more likely to elect CPM than patients who did not undergo mastectomy (odds ratio [OR] 51.267, 95\% confidence interval [95\%CI] 2.879-912.989, $p=0.0074$,Table 3).

TABLE 1. Characteristics of the 100 patients with DCIS who tested negative for BRCA mutation.

\begin{tabular}{|c|c|c|c|}
\hline Variable & Category & Total no. of patients & Total no. of patients ( $\%)$ \\
\hline \multirow{2}{*}{ Contralateral prophylactic mastectomy } & No & 69 & 69 \\
\hline & Yes & 31 & 31 \\
\hline \multirow{2}{*}{ Age at diagnosis } & $\leq 42$ years & 52 & 52 \\
\hline & $>42$ years & 48 & 48 \\
\hline \multirow{5}{*}{ Ashkenazi Jewish ethnicity(AJ) } & No & 89 & 89 \\
\hline & Unknown & 2 & 2 \\
\hline & Yes, unspecified & 1 & 1 \\
\hline & Yes, paternal side & 3 & 3 \\
\hline & Yes, both sides & 5 & 5 \\
\hline \multirow{5}{*}{ Race } & Unknown & 2 & 2 \\
\hline & AJ White & 9 & 9 \\
\hline & Black & 5 & 5 \\
\hline & Non-AJ White & 68 & 68 \\
\hline & Others & 16 & 16 \\
\hline \multirow{2}{*}{ Marital status } & Unmarried & 25 & 25 \\
\hline & Married & 75 & 75 \\
\hline \multirow{6}{*}{ Education } & Advanced degree & 24 & 24 \\
\hline & College & 31 & 31 \\
\hline & Some college/technical school & 23 & 23 \\
\hline & High school & 11 & 11 \\
\hline & Less than high school & 1 & 1 \\
\hline & Unknown & 10 & 10 \\
\hline \multirow{2}{*}{ First-degree family history of breast cancer } & 0 & 57 & 57 \\
\hline & $\geq 1$ & 43 & 43 \\
\hline \multirow{2}{*}{ Total no. of relatives with a breast cancer diagnosis } & 0 & 10 & 10 \\
\hline & $\geq 1$ & 90 & 90 \\
\hline \multirow{2}{*}{ First-degree family history of ovarian cancer } & 0 & 97 & 97 \\
\hline & $\geq 1$ & 3 & 3 \\
\hline \multirow{2}{*}{ Total no. of relatives with an ovarian cancer diagnosis } & 0 & 85 & 85 \\
\hline & $\geq 1$ & 15 & 15 \\
\hline \multirow{3}{*}{ Estrogen receptor status } & Negative & 18 & 18 \\
\hline & Positive & 66 & 66 \\
\hline & Unknown & 16 & 16 \\
\hline \multirow{3}{*}{ Progesterone receptor status } & Negative & 29 & 29 \\
\hline & Positive & 54 & 54 \\
\hline & Unknown & 17 & 17 \\
\hline \multirow{4}{*}{ Nuclear grade } & $\mathrm{I}$ & 8 & 8 \\
\hline & II & 37 & 37 \\
\hline & III & 44 & 44 \\
\hline & Unknown & 11 & 11 \\
\hline \multirow{3}{*}{ Mastectomy of affected breast } & No & 31 & 31 \\
\hline & Yes & 67 & 67 \\
\hline & Unknown & 2 & 2 \\
\hline \multirow{5}{*}{ Mastectomy-Reconstruction } & Delayed & 7 & 7 \\
\hline & Immediate & 50 & 50 \\
\hline & No Mastectomy & 31 & 31 \\
\hline & No reconstruction & 10 & 10 \\
\hline & Unknown & 2 & 2 \\
\hline \multirow{3}{*}{ Tamoxifen Treatment } & No & 77 & 77 \\
\hline & Yes & 20 & 20 \\
\hline & Unknown & 3 & 3 \\
\hline
\end{tabular}


TABLE 2. Association between patient characteristics and contralateral prophylactic mastectomy (CPM) in the univariate analysis.

\begin{tabular}{|c|c|c|c|c|}
\hline Covariate & Variables & CPM (\%) & No CPM (\%) & $p$ value \\
\hline \multirow{2}{*}{ Age at diagnosis } & $\leq 42$ years & $19(36.5 \%)$ & $33(63.5 \%)$ & \multirow[t]{2}{*}{0.2126} \\
\hline & $>42$ years & $12(25 \%)$ & $36(75 \%)$ & \\
\hline \multirow{4}{*}{ Race } & Ashkenazi Jewish White & $5(55.6 \%)$ & $4(44.4 \%)$ & \multirow[t]{4}{*}{0.1402} \\
\hline & Non-Ashkenazi Jewish White & $21(30.9 \%)$ & $47(69.1 \%)$ & \\
\hline & Black & $2(40 \%)$ & $3(60 \%)$ & \\
\hline & Other & $2(12.5 \%)$ & $14(87.5 \%)$ & \\
\hline \multirow{2}{*}{ Marital status } & Not married & $3(12 \%)$ & $22(88 \%)$ & \multirow[t]{2}{*}{0.0235} \\
\hline & Married & $28(37.3 \%)$ & $47(62.7 \%)$ & \\
\hline \multirow{3}{*}{ Education } & Advanced degree & $6(25 \%)$ & $18(75 \%)$ & \multirow[t]{3}{*}{0.7543} \\
\hline & College/some college or technical school & $18(33.3 \%)$ & $36(66.7 \%)$ & \\
\hline & High school or less & $3(25 \%)$ & $9(75 \%)$ & \\
\hline \multirow{2}{*}{$\begin{array}{l}\text { First-degree family history of } \\
\text { breast cancer }\end{array}$} & 0 & $15(26.3 \%)$ & $42(73.7 \%)$ & \multirow[t]{2}{*}{0.2436} \\
\hline & $\geq 1$ & $16(37.2 \%)$ & $27(62.8 \%)$ & \\
\hline \multirow{2}{*}{$\begin{array}{l}\text { Total no. of relatives with a breast } \\
\text { cancer diagnosis }\end{array}$} & 0 & $2(20 \%)$ & $8(80 \%)$ & \multirow[t]{2}{*}{0.7198} \\
\hline & $\geq 1$ & $29(32.2 \%)$ & $61(67.8 \%)$ & \\
\hline \multirow{2}{*}{$\begin{array}{l}\text { First-degree family history of } \\
\text { ovarian cancer }\end{array}$} & 0 & $28(28.9 \%)$ & $69(71.1 \%)$ & \multirow[t]{2}{*}{0.0278} \\
\hline & $\geq 1$ & $3(100 \%)$ & $0(0 \%)$ & \\
\hline \multirow{2}{*}{$\begin{array}{l}\text { Total no. of relatives with an ovar- } \\
\text { ian cancer diagnosis }\end{array}$} & 0 & $23(27.1 \%)$ & $62(72.9 \%)$ & \multirow[t]{2}{*}{0.0425} \\
\hline & $\geq 1$ & $8(53.3 \%)$ & $7(46.7 \%)$ & \\
\hline \multirow{2}{*}{ Estrogen receptor status } & Negative & $5(27.8 \%)$ & $13(72.2 \%)$ & \multirow[t]{2}{*}{1.000} \\
\hline & Positive & $19(28.8 \%)$ & $47(71.2 \%)$ & \\
\hline \multirow{2}{*}{ Progesterone receptor status } & Negative & $7(24.1 \%)$ & $22(75.9 \%)$ & \multirow[t]{2}{*}{0.4817} \\
\hline & Positive & $17(31.5 \%)$ & $37(68.5 \%)$ & \\
\hline \multirow{3}{*}{ Nuclear grade } & $\mathrm{I}$ & $4(50 \%)$ & $4(50 \%)$ & \multirow[t]{3}{*}{0.3341} \\
\hline & II & $9(24.3 \%)$ & $28(75.7 \%)$ & \\
\hline & III & $14(31.8 \%)$ & $30(68.2 \%)$ & \\
\hline \multirow{2}{*}{ Mastectomy } & No & $0(0 \%)$ & $31(100 \%)$ & \multirow[t]{2}{*}{$<0.0001$} \\
\hline & Yes & $30(44.8 \%)$ & $37(55.2 \%)$ & \\
\hline \multirow{4}{*}{ Mastectomy and reconstruction } & Delayed (total mastectomy + delayed reconstruction) & $6(85.7 \%)$ & $1(14.3 \%)$ & \multirow[t]{4}{*}{$<0.0001$} \\
\hline & Immediate (total mastectomy + Immediate reconstruction) & $19(38 \%)$ & $31(62 \%)$ & \\
\hline & No mastectomy (partial mastectomy + no reconstruction) & $0(0 \%)$ & $31(100 \%)$ & \\
\hline & No reconstruction (total mastectomy + no reconstruction) & $5(50 \%)$ & $5(50 \%)$ & \\
\hline \multirow{2}{*}{ Tamoxifen Treatment } & No & $29(37.7 \%)$ & $48(62.3 \%)$ & \multirow[t]{2}{*}{0.0054} \\
\hline & Yes & $1(5 \%)$ & $19(95 \%)$ & \\
\hline
\end{tabular}

TABLE 3. Association between patient characteristics and contralateral prophylactic mastectomy in the multivariate analysis.

\begin{tabular}{|c|c|c|c|c|c|}
\hline \multirow{2}{*}{\multicolumn{2}{|c|}{ Parameter }} & \multirow{2}{*}{$\begin{array}{l}p \\
\text { Value }\end{array}$} & \multirow{2}{*}{$\begin{array}{l}\text { Odds ratio } \\
\text { (OR) }\end{array}$} & \multicolumn{2}{|c|}{$95 \%$ CI of OR } \\
\hline & & & & $\begin{array}{l}\text { Lower } \\
\text { limit }\end{array}$ & $\begin{array}{l}\text { Upper } \\
\text { limit }\end{array}$ \\
\hline Mastectomy & $\begin{array}{l}\text { Yes vs. } \\
\text { No }\end{array}$ & 0.0074 & 51.267 & 2.879 & 912.989 \\
\hline
\end{tabular}

\section{Discussion}

Our study found a 31\% CPM election rate among 100 DCIS patients who tested negative for a BRCA mutation. Factors associated with CPM in this cohort were marital status, family history of $\mathrm{OC}$, reconstruction, mastectomy of the affected breast, and tamoxifen use. To our knowledge, our study uniquely examines CPM rates among DCIS-only patients who are BRCA negative.

Our recent previous study examined CPM among patients with DCIS who were either $B R C A$-positive, BRCA-negative, or untested. ${ }^{3}$ In that study, 165 patients $(27 \%)$ elected CPM. More specifically, CPM was elected by $12(71 \%)$ of 17
$B R C A$-positive patients, 23 (25\%) of 91 BRCA-negative patients, and $9(16 \%)$ of the 57 patients who did not undergo genetic testing. Factors associated with CPM in that study were age $\leq 45$ years, family history of OC, and BRCA positivity. The current study included only $B R C A$-negative patients, and the results demonstrated some findings distinguishable from those of our previous studies. Specifically, age ( $<45$ years) was significantly associated with CPM election in the previous article; however, in the current study, while the trend remained as before, age was not a significant predictor of CPM. Marital status was a significant predictor in the current study but not in the previous one.

It is possible that current results differed slightly from previous results due to differences in the analyzed patient cohort, such as BRCA testing status and results. Both studies, however, found that family history of OC predicts CPM among patients with DCIS, regardless of $B R C A$ results. $B R C A$ positivity is known to be associated with significantly increased risks for $\mathrm{BC}$ and $\mathrm{CBC},{ }^{14}$ which may contribute to $\mathrm{CPM}$ election among $B R C A$-positive patients. 
However, our current results suggest that family history of OC is associated with CPM even when patients are $B R C A$-negative. More specifically, a family history of $\geq 1$ relatives with OC was significantly associated with increased rates of CPM election. To our knowledge, this is the first study to report an association between family history of OC and CPM election among BRCA-negative patients.

This significant finding poses the question of why a family history of $\mathrm{OC}$ is associated with increased CPM rates, particularly in BRCA-negative women. It is possible that the low survival rate of women with OC may have contributed to the perspective on cancer of women in the current study, and thus this group was more willing to undergo preventive surgery to reduce chances of future cancer. Katapodi et al. ${ }^{15}$ observed that most women at high risk for $\mathrm{BC}$ underestimated their actual risk. Individuals with a family history of $\mathrm{BC}$ may have a more skewed perspective if their family members with $B C$ survived, and this may contribute to the lack of significance of a family history of BC related to CPM election. Future studies are needed to analyze the effect of cancer deaths in families to verify this hypothesis.

According to Musiello and associates, ${ }^{16}$ surgeons reported fear of developing another $\mathrm{BC}$ and a desire for breast symmetry as among the top reasons patients opted for CPM. Abbott et al. ${ }^{17}$ found that women with BC, including DCIS, substantially overestimated their risk for developing $\mathrm{CBC}$. In the presence of a negative $B R C A$ test result, the experience of a personal $\mathrm{BC}$ diagnosis may trigger fear and anxiety concerning future risk of $\mathrm{CBC}$ and thus may lead to $\mathrm{CPM}$ election. An individual patient's concerns regarding the risk of $\mathrm{CBC}$ may be particularly warranted when there is a strong family history of breast cancer, which may be due to an unidentifiable hereditary cause, other than the BRCA genes. Thus, even though the patient is $B R C A$-negative, the decision to proceed with CPM may be appropriate due to concern about an unexplained family history. Furthermore a diagnosis of DCIS increases the risk for invasive cancer and $\mathrm{CBC}$ by $0.5 \%$ to $1 \%$ per year regardless of family history $1,2,8$; therefore the reported statistics of additional cancer risk may also contribute to the rate of CPM among women with DCIS.

The experience of frequent screening, the potential need for subsequent biopsies, and the worry and fear of anticipating test results are emotionally exhausting and may lead many women to undergo preventive surgeries. Many patients may feel burnout due to frequent cancer surveillance, and this may contribute to their desire for acceptance of CPM. Hoskin and Greene ${ }^{18}$ indicated that preventive mas- tectomies could be a result of screening fatigue and/or stressful and costly screening. Future prospective study is needed to determine the significance of these factors in this cohort.

The various studies discussed here, including the results of our analysis, suggest that a personal history of $\mathrm{BC}$ and/or family history of OC may be sufficient to prompt the decision to undergo CPM despite a negative $B R C A$ result. Subjective factors such as anxiety and fear, along with objective factors such as a family history of OC, being married, and choosing mastectomy, may explain the relatively high rate of CPM reported in this study.

It has been previously reported that marriage affects the decision to undergo CPM among DCIS patients. Howard-McNatt et al. ${ }^{11}$ examined CPM among patients with invasive $B C$ who tested negative for a $B R C A$ mutation; among 110 women who underwent genetic testing, $37 \%$ of the $B R C A$-negative women chose CPM. Married women in that study were significantly more likely to elect CPM than those who were not married. This finding is consistent with our finding that marital status is a significant predictor of CPM. Married women may have emotional support from their partner, as well as an established relationship; thus their concerns related to body image may be less impactful than for single women. Further studies are needed to define more specifically the factors related to CPM election among married women.

The decision to proceed with mastectomy of the affected breast increases the rate of CPM. ${ }^{2,} 19$ In this cohort, patients who had mastectomy were significantly more likely to elect CPM than those who had only breast-conserving surgery. Tuttle and associates ${ }^{2}$ analyzed the initial treatment of patients with unilateral DCIS between 1998 and 2005 to determine the CPM rate. A 13.5\% CPM rate was found for patients who had a mastectomy on the affected side. In addition, Yao et al. ${ }^{20}$ observed a universal increase in CPM among surgically treated patients with invasive and noninvasive BC. Among 23,218 surgically treated patients who elected CPM, election increased from $0.4 \%$ in 1998 to $4.7 \%$ in 2007.

Our study also indicated increased use of reconstruction among patients with DCIS. Recent research has shown a similar rapid increase. ${ }^{21}$ The past two decades have witnessed increasing advances in reconstruction techniques and minimizing mastectomy scars, which may appeal to patients considering options for managing their cancers. There is a known association between reconstruction and an increased CPM rate among women who have unilateral mastectomy for DCIS. 22,23

Previous work reported that the use of tamoxifen 
reduces the 5-year rate of ipsilateral and contralateral $\mathrm{BC}$ in patients with DCIS; factors predicting tamoxifen use in their study were breast-conserving surgery and younger age. ${ }^{24}$ Consistent with their conclusion, our study showed that patients who took tamoxifen were less likely to elect CPM than those who did not. Future studies are needed to verify this finding.

Limitations of this study include the fact that this cohort included only patients referred for genetic counseling and testing. Therefore, they were considered to have a higher baseline risk. We recognize the findings of this study are derived from a small sample, and are specific to BRCA-negative patients; therefore, may not be generalized to all DCIS patients. Moreover, given the uneven distribution of our groups limits our findings and their generalizability to a larger sample with a more even distribution. Additionally, future prospective studies are needed to evaluate the complex decision-making processes leading to CPM despite negative BRCA results. A questionnaire or interview-based study could further elucidate which specific factors consciously affect patients' decision for CPM.

The present study indicated a relatively high CPM rate among DCIS patients with negative $B R C A$ results which has not been clearly established among this specific cohort. Our analyses highlight not only the increasing trend of CPM among patients with DCIS, but also the different predictive factors among $B R C A$ carriers vs. non-carriers. The predictors shown in this BRCA-negative cohort may need to be considered during discussions regarding surgical decision-making. Our results highlight the need for a more individualized and focused recommendation for this group.

\section{Acknowledgments}

This study was partially funded by the Lynne Cohen Foundation for Ovarian Cancer Research, the Nellie B. Connally Breast Cancer Research Fund, the University of Texas MD Anderson Cancer Center Support Grant (CA016672) from the National Institutes of Health, and IBC Foundation.

\section{Conflict of Interests}

The authors have no financial disclosures to declare.

\section{References}

1. Kuerer HM, Albarracin CT, Yang WT, et al. Ductal carcinoma in situ: state of the science and roadmap to advance the field. J Clin Oncol. 2009; 27:279-88.

2. Tuttle $\mathrm{T}$, Jarosek $\mathrm{S}$, Habermann $\mathrm{E}$, et al. Increasing rates of contralateral prophylactic mastectomy among patients with ductal carcinoma in situ. J Clin Oncol. 2009; 27:1362-7

3. Elsayegh N, Kuerer HM, Lin H, et al. Predictors that influence contralateral prophylactic mastectomy election among women with ductal carcinoma in situ who were evaluated for BRCA genetic testing. Ann Surg Oncol. 2014; 21:3466-72.
4. Soran A, Kamali Polat A, Johnson R, McGuire KP. Increasing trend of contralateral prophylactic mastectomy: what are the factors behind this phenomenon? Surgeon. 2014; 12:316-22.

5. Ashfaq A, McGhan LJ, Pockaj BA, et al. Impact of breast reconstruction on the decision to undergo contralateral prophylactic mastectomy. Ann Surg Oncol. 2014; 14:3712-8.

6. Ford D, Easton DF, Stratton M, et al. Genetic heterogeneity and penetrance analysis of the BRCA1 and BRCA2 genes in breast cancer families. The Breast Cancer Linkage Consortium. Am J Hum Genet. 1998; 62:676-89.

7. Chen S, Iversen ES, Friebel T, et al. Characterization of BRCA1 and BRCA2 mutations in a large United States sample. J Clin Oncol. 2006; 24:863-71.

8. Bayraktar S, Elsayegh N, Gutierrez Barrera AM, et al. Predictive factors for BRCA1/BRCA2 mutations in women with ductal carcinoma in situ. Cancer. 2012; 118:1515-22.

9. Hwang ES, McLennan JL, Moore DH, Crawford BB, Esserman LJ, Ziegler JL. Ductal carcinoma in situ in BRCA mutation carriers. J Clin Oncol. 2007; 25:642-7.

10. Metcalfe KA, Finch A, Poll A, et al. Breast cancer risk in women with a family history of breast or ovarian cancer who have tested negative for a BRCA1 or BRCA2 mutation. Br J Cancer. 2009; 100:421-5.

11. Howard-McNatt M, Schroll RW, Hurt GJ, Levine EA. Contralateral prophylactic mastectomy in breast cancer patients who test negative for BRCA mutation. The Am J Surg. 2011; 202:298-302.

12. Snedecor GW, Cochran WG. Statistical Methods, 7th ed. Ames, IA: The Iowa State University Press, 1980.

13. Hosmer DW, Lemeshow S. Applied Logistic Regression, 2nd ed. New York: Wiley, 2000.

14. Stuckey A, Dizon D, Scalia Wilbur J, Kent J, Tejada-Berges T, Gass J, Legare R. Clinical characteristics and choices regarding risk-reducing surgery in BRCA mutation carriers. Gynecol Obstet Invest. 2010; 69:270-3.

15. Katapodi MC, Dodd MJ, Lee KA, Facione NC. Underestimation of breast cancer risk: influence on screening behavior. Oncol Nurs Forum. 2009; 36:306-14.

16. Musiello T, Bornhammer E, Saunders C. Breast surgeon's perceptions and attitudes towards contralateral prophylactic mastectomy. ANZ J Surg. 2013; 83:527-32.

17. Abbott A, Rueth N, Pappas-Varco S, Kuntz K, Kerr E, Tuttle T. Perceptions of contralateral breast cancer: an overestimation of risk. Ann Surg Oncol. 2011; 18:3129-36.

18. Hoskins LM, Greene MH. Anticipatory loss and early mastectomy for young female BRCA1/2 carriers. Qual Health Res. 2012; 22:1633-46

19. Tunon-de-Lara C, André G, Macgrogan G, et al. Ductal carcinoma in situ of the breast: influence of age on diagnostic, therapeutic, and prognostic features. Retrospective study of 812 patients. Ann Surg Oncol. 2011; 18:1372-9.

20. Yao K, Stewart AK, Winchester DJ, Winchester DP. Trends in contralateral prophylactic mastectomy for unilateral cancer: a report from the National Cancer Data Base, 1998-2007. Ann Surg Oncol. 2010; 17:2554-62.

21. Jagsi R, Jiang J, Momoh AO, et al. Trends and variation in use of breast reconstruction in patients with breast cancer undergoing mastectomy in the United States. J Clin Oncol. 2014; 32:919-26.

22. Kronowitz SJ, Kuerer HM. Advances and surgical decision-making for breast reconstruction. Cancer. 2006; 107:893-907.

23. Alvarado R, Lari SA, Roses RE, et al. Biology, treatment, and outcome in very young and older women with DCIS. Ann Surg Oncol. 2012; 19:3777-84.

24. Yen TWF, Kuerer HM, Ottesen RA, et al. Impact of randomized trial results in the National Comprehensive Cancer Network on the use of tamoxifen after breast surgery for ductal carcinoma in situ. J Clin Oncol. 2007; 25:3251-8. 